\title{
Integration of Cyber-Physical Systems in E- Science Environment: State-of-the-Art, Problems and Effective Solutions
}

\author{
Tahmasib Kh. Fataliyev* \\ Institute of Information Technology of ANAS, Baku, Azerbaijan \\ Email: *tfataliyev@gmail.com \\ Shakir A. Mehdiyev \\ Institute of Information Technology of ANAS, Baku, Azerbaijan \\ Email: depart11@iit.science.az
}

Received: 24 June 2019; Accepted: 14 August 2019; Published: 08 September 2019

\begin{abstract}
The implementation of the concept of building an information society implies a widespread introduction of IT in all areas of modern society, including in the field of science. Here, the further progressive development and deepening of scientific research and connections presuppose a special role of e-science. E-science is closely connected with the innovative potential of IT, including the Internet technologies, the Internet of things, cyber-physical systems, which provide the means and solutions to the problems associated with the collection of scientific data, their storage, processing, and transmission. The integration of cyber-physical systems is accompanied by the exponential growth of scientific data that require professional management, analysis for the acquisition of new knowledge and the qualitative development of science. In the framework of e-science, cloud technologies are now widely used, which represent a centralized infrastructure with its inherent characteristic that is associated with an increase in the number of connected devices and the generation of scientific data. This ultimately leads to a conflict of resources, an increase in processing delay, losses, and the adoption of ineffective decisions. The article is devoted to the analysis of the current state and problems of integration of cyber-physical systems in the environment of e-science and ways to effectively solve key problems. The environment of e-science is considered in the context of a smart city. It presents the possibilities of using the cloud, fog, dew computing, and blockchain technologies, as well as a technological solution for decentralized processing of scientific data.
\end{abstract}

Index Terms-E-science, cyber-physical systems, integration, big scientific data, smart city, cloud computing, blockchain.

\section{INTRODUCTION}

The term electronic science (e-science), introduced in 1999 by Dr. John Taylor, then the director of the UK's scientific councils, combines revolutionary methods of conducting collective experimental research in networked research infrastructure. This infrastructure has allowed scientists to use technical resources in a coordinated way, which are usually distributed, maintained by various organizations and belong to different scientific disciplines, and simplifies the use and access to basic research tools, such as computing resources and databases. E-science, providing modern solutions in the areas of online education, virtual laboratories, global research networks, in computer tools, etc., helps and continues to help make rapid progress in science. Modern digital technologies form new partners in science, such as cyberinfrastructure, e-science, civil science, Data science, Big data. Digital science, in turn, has led to a significant increase in the volume of scientific data as one of the main trends in the development of science.

Further development of information technologies has generated such new paradigms as the Internet of things (IoT), cyber-physical systems (CPS), industry 4.0, cloud computing, blockchain, etc., which have brought and will bring many advantages and potential opportunities in the structure of e-science. Modern ideas of automation in current research projects are also based on CPS, used as intelligent control systems.

A wide range of CPS applications includes transportation, agriculture, healthcare, aerospace, etc. Science is one of its interesting applications. The integration of this system into the e-science environment leads to multiple increases in the flow of scientific data. As a result, the problems of organizing and processing large scientific data become relevant along with other [1]. 
CPS is especially focused on accurate measurement, storage, processing, analysis and presentation of data. Here there is a problem of archiving information, on the one hand, and, on the other hand, it is necessary to organize, distribute and provide the information requested by the user in the information retrieval service. Along with this, the discovery of hidden knowledge from the collected scientific data is also relevant and important.

Thus, the solution of problems with big data is important for the modern digital age. The article explores the problems, reveals the prospects for the development of technological solutions for processing scientific data generated from the integration of CPS in the e-science environment.

\section{Preconditions for CREAting CPS}

The work of CPS is based on the principle of integration of computational and physical processes, that is, it is part of a system of physical objects, and the term CPS was coined in 2006 by the US National Science Foundation [2]. CPS is a system that consists of various subsystems in which control at the physical level is carried out on the basis of processing signals from multiple sensors and making decisions at the cyber level $[3,4]$. In [5] introduces a new analysis framework for classifying Cyber-Physical Production Systems applications relatively to various items, including their cognitive abilities, their application extent, the interaction with human operators, the distribution of intelligence and the network technologies that are used. CPS's are defined as systems with decentralized control, resulting from a merger between the real and virtual worlds, having autonomous behavior and depending on the context in which they are located. They are capable of forming complex hierarchical CPS, where deep cooperation with a person is also assumed. For this, embedded software in CPS uses sensors and actuators, connect to each other and to human operators by communicating via interfaces and have storage and data processing capabilities from the sensors or the network.

From a technical point of view, CPS is a system implemented via the IoT, Industry 4.0, Industrial Internet of Things (IIoT), Machine-to-Machine Interaction (M2M), wireless sensor networks (WSN), cloud computing. Essentially, WSN, M2M, IoT, and CPS are made up of similar components. Both IoT and CPS are aimed at expanding the connection between the cyberspace and the physical world through information perception and interactive technologies. But they have obvious differences: IoT focuses on the network and aims to unite all things in the physical world. Thus, it is an open network platform and infrastructure. CPS emphasizes the exchange of information and feedback, where the system should control the physical world in addition to the perception of the physical world, forming a closed system [6].

The similar aspects of these technologies complement each other and extend their functionality:
- WSN, M2M, and CPS belong to IoT.

- WSN is the basic IoT and CPS scenario.

- WSNs regarded as the supplement of M2M is the foundation of CPS.

- CPS is an evolution of M2M in intelligent information processing.

- M2M is the main IoT model at the present stage.

- CPS will be an important technical form for IoT in the future [7].

Wireless technologies such as Bluetooth, Wi-Fi, ZigBee, LoRa, etc. allow you to directly extract information from sensors installed in previously inaccessible areas for measuring parameters of various technological processes [8,9]. In this context, a significant role belongs to such network technologies as cloud, fog and dew computing, which help to store large amounts of information and allow the use of complex analytical tools such as big data, data mining, OLAP, pattern recognition, etc. [10].

As you know, the emergence of CPS has become possible because of the rapid development of information technology. The expansion of the coverage of the Internet, its availability, the emergence of progressive IPv6 technology, which removed the restrictions on the number of connected sensors and devices, as well as the emergence of functionally new primary information mini, micro and nanosensors created comprehensive technical capabilities for monitoring and managing physical processes, experiments and production directly via the Internet and became the basis for integrating CPS into these processes.

\section{RELATED WORKS}

Analysis of the published works shows that due to the unique functions of the CPS, technical solutions on their platform are not limited to specific areas. In ref. [11] shows common applications of CPS, among which in our case the most interesting of them are discussed further.

CPS in transport is an integrated transportation management system aimed at achieving safer and more intelligent traffic [12]. This system collects, processes, analyzes and visualizes data from sensors located on mobile devices. The result of this CPS can be the optimization of traffic, monitoring of road surface, detection of hazards, automotive networks and so on.

CPS in medicine is a classic example of closed-loop feedback control systems. Application scenarios for such CPSs vary from patient monitoring, analgesic infusion pumps to implantation of sensory devices [13]. Any change of an object in the physical world can be directly modeled and improved on its counterpart in the cyber world, and in the physical world actions will be taken based on instructions from cyberspace

CPS to control wind turbines [14] is used to reduce energy costs and increase profits. Data from wind generators tend to be very large, and therefore, to dynamically represent the behavior of CPS, instead of 
traditional statistical data analysis methods, genetic algorithms are used.

Integration of CPS in the library can improve the quality and quantity of traditional library services, for example, intellectual inventory, intellectual inquiry, selfemployment and self-return, searching for inappropriate or incorrectly delivered books or materials, automatically combating counterfeit products, providing contextual prompts and information, signaling the presence tools and resources, streamlining internal library processes. The CPS will also be able to control temperature and humidity, energy consumption, fire safety, eliminate hidden security risks, create comfortable conditions for both visitors and for the preservation of ancient manuscripts and valuable books [15].

It should be noted that there are examples of the integration of CPS for solving problems and tasks in the field of science.

In the field of earth sciences, there is CPS for monitoring volcanic activity. It is designed to collect data from remote sensors in the WSN. Based on the collected data, they are processed to further analyze and monitor the hazards by assessing the level of volcanic unrest and understanding the physical processes occurring in the volcano, such as mechanisms of migration and magma eruption [16].

Ref. [17] describes CPS for environmental monitoring, which collects large multi-dimensional and multitemporal data from the global atmosphere. For these purposes, space and aviation sensors are used for remote observation of the Earth and measure background radiation. This is the physical level of the system. At the cyber level, special technologies are used to process and interpret data, which make it possible to obtain images of the earth's surface. Later, on the basis of these images, traditional or thematic maps, resource summaries, etc. are developed. Then, at the level of data analysis, decisions or recommendations are made for further actions in certain areas of activity.

Also known CPS, that monitors the environmental conditions or the ambient conditions in indoor spaces at remote locations. The communication between the system's components is performed using the existent wireless infrastructure based on the IEEE $802.11 \mathrm{~b} / \mathrm{g}$ standards. The resulted solution provides the possibility of logging measurements from locations all over the world and of visualizing and analyzing the gathered data from any device connected to the Internet [18].

A known system of adaptive control of a radio telescope on the platform of the CPS. Adaptive control is carried out on the basis of preliminary calculations of data received from sensors. In this case, it is important to provide high computational performance, because otherwise, the reaction time may be too long. [19].

There are currently available solutions with the use of various well-known structures for the creation of remote laboratories with automation technologies. These solutions provide IoT structures that can be used to build and operate functional systems in a web browser for different areas. They can also be considered as a platform for the integration of CPSs in the creation of virtual laboratories.

In ref. [20] discusses the IIoT Web-Oriented Automation System (WOAS) platform, a prototype of a web platform that allows the integration of CPS services, including components of distributed devices, into a functional system. In this functional system, it is not necessary to have a technical process automation system or a remote scientific or academic laboratory. The IIoT WOAS platform allows you to fully configure and use browser-based functional systems consisting of technical devices and systems, such as CPS components and related services. This platform was designed to use automation technologies and can also be used to create and operate a laboratory for remote experiments over the Internet using technical equipment and systems. As a rule, here the type of technical devices does not matter. The only requirement is that the device is connected to the Internet as a component of CPS and be available. Many user-oriented WOAS portals allow you to create and manage a virtually unlimited number of virtual laboratories.

To ensure the sustainable functioning of the e-science infrastructure, a complex of measures is needed to solve the problems arising in it. To support decision making at this level, the organization of maintenance can provide substantial assistance in maintaining system safety, reducing failure rates and preventing malfunctions. In ref. [21] analyzes the performance of the electronic scientific infrastructure as CPS, presents its conceptual model, addresses the problems of ensuring its security and the creation of electronic maintenance.

The integration of CPS into the e-science environment also provides a wide range of opportunities for the implementation of interdisciplinary research principles. As an example, we can consider bioinformatics as an interdisciplinary activity in cyber-physical space. It is known that bioinformatics combines computer science, statistics and mathematical methods for analyzing and interpreting biological data. Here, the integration of CPS here leads to the automatic execution of various biological analyzes, which are very difficult to carry out manually, an exponential increase in data analysis and the accuracy of the results [22].

Virtual Observatory (VO) may be another example of CPS in science. VO is a collection of interoperable data archives, tools, and applications that together form an environment in which original astronomical research can be carried out. The VO is opening up new ways of exploiting the huge amount of data provided by the evergrowing number of ground-based and space facilities, as well as by computer simulations [23].

Ref. [24] includes the complete solution of CPS, beginning with physical level, comprising from claiming sensors, processor and the correspondence protocol, and arriving at information management and stockpiling at the digital level. The test outcomes indicate that the suggested framework represents a feasible and straightforward solution for economical monitoring applications. 
An important component of the e-science cyberinfrastructure is the Datacenter, which must be immune to incidents and unforeseen circumstances causing system failures. The Datacenter can also be represented in the aspect of CPS, in which the management of IT and cooling technologies in them are classified according to the degree to which they take into account both cyber-physical and physical factors [25].

Thus, the questions raised in the article are of great interest because of their relevance. A comprehensive solution to them in a single environment requires continuation of research and the development of effective methods.

\section{PRoblems INTEGRATION OF CPS TO E-SCIENCE ENVIRONMENT}

Based on the studies in the previous sections, it can be concluded that cyber-physical integration in scientific research can be conducted in the following aspects.

\section{A. Integration of CPS into the e-science infrastructure}

Unlike the traditional definition, we consider e-science in a broader sense. This implies the introduction of ICT in all areas of research enterprises and organizations, including management.

The basis of e-science is physical infrastructure, which may include telecommunications networks, data centers, research jobs, research laboratories, buildings, electricity, logistics, etc.

This physical infrastructure can be viewed in the context of integrating CPS on the smart city platform. Definitions of a smart city are interpreted in different variations. A smart city is used throughout the world under different names and in different circumstances, and therefore there are a number of conceptual options created by replacing smart adjectives with other alternative adjectives [26,27]. In general, the concept of the smart city implies widespread informatization, which implies the presence of a multitude of sensors for retrieving information, primary devices for collecting, processing and storing data, intelligent analytics and the presence of smart inhabitants (in our case, these are escientists), interested in applying the above solutions.

The technological infrastructure of the smart city is a platform of CPS, which can be applied to the infrastructure of the National Academy of Sciences of Azerbaijan (ANAS) (Fig. 1).

From Fig. 1. it follows that the structure of ANAS unites six scientific divisions. In turn, specialized research institutes function in the structure of these divisions. In our approach, this structure is usually perceived as a smart city, and units - smart areas and research institutions - as smart buildings.

As follows from Fig. 1, the scope of a smart city is ANAS. Further, at the level of a smart area, there are scientific units. As for specialized agencies, they are under the influence of a smart building.

CPS in such a smart structure, both globally and locally, can solve their following problems:
- Uninterrupted power supply;

- Materials and equipment management;

- Equipment monitoring;

- Maintenance;

- Building security;

- Video observation;

- Detection and warning of danger;

- User identification;

- Tracking and identification of hazardous materials;

- Environmental monitoring;

- Creating a comfortable working environment for researchers;

- Climate control;

- Waste management, etc.

For example, in [28] it is shown that a modern building automation system collects data from temperature and humidity values to the state of the engine and often includes possibilities for optimizing energy consumption. That is, with the optimal start/stop, the building automation system will know when it should turn on the air conditioning system for a specific area in the building.

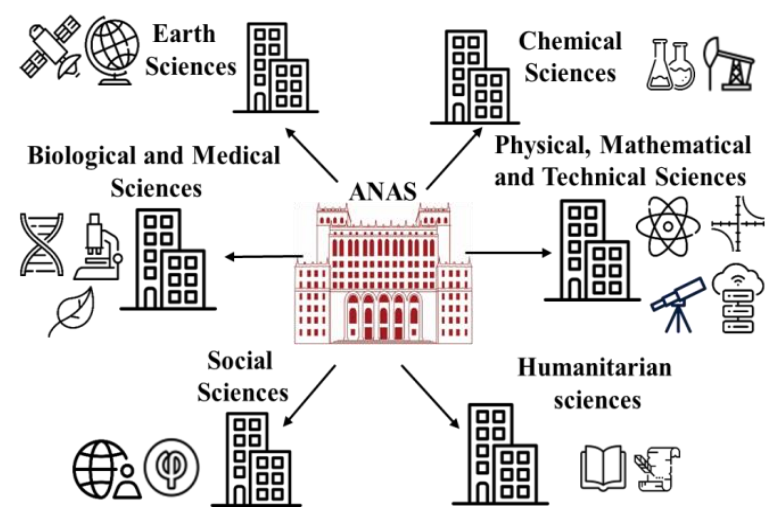

Fig.1. Considering the integration of CPS into the e-science environment like a smart city.

\section{B. Integration of CPS into the e-science research environment}

Further, in the context of integrating CPS into the research environment of e-science, its generalized architecture consists of several levels. Their characteristics are listed below:

- At the level of physical objects, data is collected from sensors installed to measure various physical parameters.

- On the cyber platform and computing level, data is mainly processed and converted into operational information to obtain information about the performance of individual components or feedback signals are generated.

- At the CPS application level, complex calculations are performed based on data processed at lower levels, and various types of physical object models are created.

- The big data analysis level is performed on a compute node, such as the cloud. At this level, new 
knowledge is gained, feedback from cyberspace can be transferred to the physical space in order to apply corrections and preventive effects on the system.

According to the presented model, it is possible to interpret CPS integration into the e-science environment as follows. Scientific data can be data from different sensors during physical experiments and chemical experiments, biological data, results of spectral analysis, photographs from telescopes, results of sociological surveys in social sciences, historical works, documents, manuscripts, etc. These data can also be transmitted to the remote units, virtual collectives, and laboratories of academics. Collected data is processed and converted to new knowledge. At the next levels, a full view of scientific research (physical events, chemical reactions, matter structure, historical event, etc.) is made. Later, the scientific community gets acquainted with the research results. Therefore, as a result of the integration of CPS to e-science environment, events take place starting from the research stage, experiment conduction, processing of obtained data to an acquaintance of scientific community with proposed theory, hypothesis or scientific recommendations, repetition and accurate results. The territorial distribution of multidisciplinary research and interdependence of the heterogeneous devices used should be taken into account. Each device can be used within the IoT concept and can be fully managed with web technologies.

Based on the above, a five level architecture of tasks related to the integration of CPS in the e-science environment is proposed (Fig. 2).

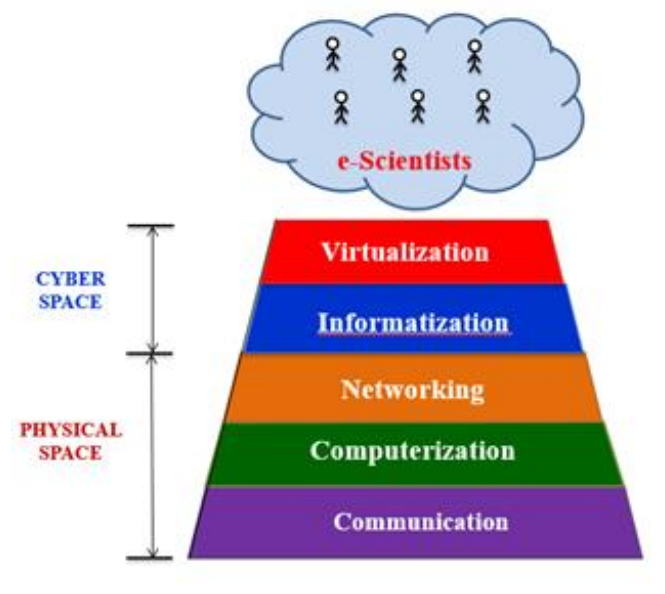

Fig.2. Five level task architecture

As noted, CPS is a complex system that combines computational, communication, and physical processes. From Fig. 2 it follows that these CPS components are also present in the architecture of e-science, which is essential in solving integration problems.

\section{Processing OF SCIENTIFIC DATA}

E-science plays a special role in the development and expansion of scientific research and connections. It covers all stages of solving problems in the research process, including the creation, collection, search, storage, processing, and analysis of scientific data, as well as science management issues. Existing IT for these purposes has created ample opportunity. In addition, the exponential growth of scientific data requires professional management of them as an essential condition for the acquisition of new knowledge and the rapid development of science. For these purposes, the Internet infrastructure is used, through which users get remote access to large-scale information and more efficiently use their computing resources. One of the main problems in the e-science environment is the problem of big data. When considering e-science as a single system; we see that it solves problems from different subsystems. Information support of science, scientometric analysis, intelligent analysis, and scientific data lead to the generation of big data [29]. The integration of CPS into this environment also plays the role of a generator for quickly increasing big data.

It should be noted that the types, volume, frequency of use, life cycle and other characteristics of scientific data are different. The following data is especially important for research:

- Observation data - obtained from telescopes, satellites, sensor networks, demographic studies, historical information, or one-time event recording. In most cases, this data cannot be repeated and, therefore, must be saved.

- Experimental data - obtained from highproductivity decides through clinical, biomedical and pharmaceutical experiments or other controlled experiments. It is especially important to store some data that is considered inappropriate to recollect due to ethical or other reasons, such as data regarding human subjects and endangered species.

- Computing data - generated as a result of the largescale computation in a supercomputer, data centers, etc. stored for a certain period and processed through intellectual analysis technologies.

- Informational data - are used by scientific societies for different purposes. Such data include the human genome, proteins, seismology, oceanography, clinical research, endangered species data.

These scientific data categories also add big data generated from the integration of CPS into the e-science environment. To solve this problem, various methods and approaches are used.

CPS interacts with the physical system through networks, the final CPS is usually the traditional centralized closely related embedded computer system, 
which contains a large number of physical systems consisting of intelligent wireless sensor networks [30]. At the same time, CPS is a product of the integration of heterogeneous systems: these are heterogeneous distributed systems with deep integration and interaction of information systems and physical systems, which should deal with the problem of time synchronization and the spatial arrangement of various components.

\section{A. Cloud, fog, and dew computing}

In the e-science environment, data from different sources is often characterized by a lack of structuring, various formats, rapid generation and a sharp increase in volume. Processing such a data flow using existing technologies is very complex and requires new technological solutions. Studies show that cloud technologies are preferable for processing big data [31]. Cloud computing provides users with remote access to services, computing resources, and software over the Internet [32]. Cloud technologies allow us to collect and store big data, on the one hand, and, on the other hand, provide the necessary processor power for data processing. A cloud analytics service that uses statistical analysis and machine learning helps reduce big data to an acceptable size so that we can get information, test hypotheses, and draw conclusions. Data can be imported from the cloud, and users can run cloud data analysis algorithms for big data sets, after which data can be saved back to the cloud.

Nevertheless, the further development of the e-science platform is accompanied by an increase in the number of installed devices and a tendency to increase the amount of scientific data generated in this environment, which leads to an overload of the Internet infrastructure. In addition, there is a significant increase in data traffic due to the widespread use of smartphones, tablets and video streaming. Users experience a decrease in network bandwidth, which, in turn, leads to resource conflicts, increased processing delays, losses, and inefficient decision making. In some cases, it may be necessary to move large data sets between multiple clouds - for example, if a single cloud is not enough for computational purposes or if the user or employees must use several cloud resources [33].

Initially, the edge computing paradigm was proposed to effectively address the problems described. Here, the reduction of network load, as well as making more operative decisions based on the data is a key requirement and problem is solved by bringing the processing near to the data source. It's computing and memory resources are used for local storage and initial data processing. But, such periphery computing has very limited capabilities that lead to resource conflict and increase processing delays. For this reason, a new paradigm called fog computing is developed, which performed the integration of periphery clouds with cloud resources in order to eliminate all deficiencies of edge computing [34]. Thus, in contrast to processing data by directly sending data from initial devices to the central server, fog computing provides processing of data directly near to the devices and sends necessary parts to a central server; its main objective is to increase productivity by directly processing network data. First computing architecture of fog is described in [35] and here, fog level is determined as distributed intellect between the base network and sensor devices.

Fog system has relatively small computing resources (memory, processing, and storage). But resources can be increased on demand. However, the significant shortfall in clouds and fog computing is dependence on the availability of Internet access. The level of development of ICT tools and methods indicates that the most promising direction in the e-science infrastructure is the dew computing that allows access to the data without the constant use of the Internet. In this context, "Dew computing is an on-premises computer softwarehardware organization paradigm in the cloud computing environment where the on-premises computer provides functionality that is independent of cloud services and is also collaborative with cloud services. The goal of dew computing is to fully realize the potentials of on-premises computers and cloud services" [36]. Dew computing was proposed in 2015 [37]. This technology ensures that the services offered are not dependent upon accessing the Internet and has two main features: first, local computers (desktop, laptop, tablet, and smartphone) show rich micro-services that do not depend on cloud services; secondly, these services mainly collaborated with cloud services. Dew server is a small local server that keeps the speed of accessible data generated from primary computing from an Internet connection or without it, and is synchronized with cloud server with connection is available again. This architecture can be used to ensure accessibility of websites in offline mode. This system can reduce the cost of data transfer in organizations with an interrupted or limited Internet connection. Thus, abovementioned justifies effectiveness and promising outlook of separate or joint use of abovementioned technologies, in accordance with specific characteristics of solved problems, in the processing of big data, including CPS integration.

\section{B. Blockchain}

To solve the problems of decentralized data processing, you can also use the innovative technology of blockchain, which, along with the computing technologies discussed earlier, can be another integration platform of e-science [38-40]. The blockchain is a database of distributed entries, which consists of all the operations performed and is divided between network members. This database is called a distributed ledger. Each operation is stored in a distributed registry and is approved by agreement of the majority of participants. All executed operations are saved in the blockchain. Thus, the blockchain provides a decentralized model of processing operations.

Consider some of the characteristics of blockchain, which make it suitable for both CPS and IoT.

- Decentralization: network transactions are supported by various decentralized nodes. 
- Scalability: the computing power of the network increases as the number of nodes in the network increases.

- Reliability: transactions are verified and confirmed by consensus between peers.

- Security: all transactions on the Blockchain network are protected by reliable cryptography.

- Sustainability: records after reaching consensus cannot be changed or deleted.

- Autonomy: devices can communicate with each other directly since each device has its own account.

The blockchain technology is constantly evolving and can make important contributions, such as protecting the rights of authors in the e-science environment, personnel management, collective decision-making, expert assessments, and information security problems.

In ref. [41] it was shown that the blockchain technology can make scientific activity open at all stages of its implementation. As noted, research areas begin with the collection or discovery of baseline data. The results of studies conducted according to a certain method become available only at the time of publication. Everything that happens before, for example, data collection and analysis, review, etc., is not transparent. This lack of transparency leads to problems associated with reproducibility, that is, the inability of researchers to reproduce experiments to confirm the findings of scientific papers. For example, in ref. [42], the possibility of "notarial approval" of registration of research results related to the time of their generation via blockchain was investigated. This application makes it impossible to change the approved registration data, prevents their manipulation and can be used to publish research results.

Thus, on the basis of the foregoing, it can be concluded that the use of decentralized computing technologies in the processing of large scientific data obtained in various fields of science in the e-science environment should be a promising direction.

\section{PROPOSED MODEL}

Existing scientific data processing model in ANAS on AzScienceNet scientific computer network platform is implemented as a cloud structure (Fig. 3).

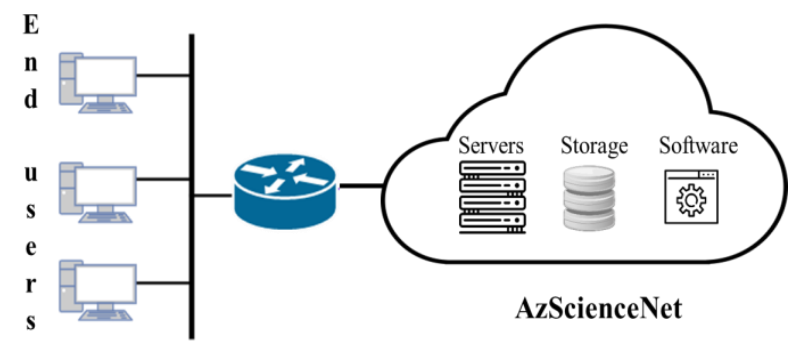

Fig.3. Existing data processing model on AzScienceNet platform
Currently, it unites about 40 scientific institutions. There are over 7000 computers and mobile devices in this infrastructure, and there is a steady upward trend in connectivity [43]. This requires an extensive development path when it is necessary to increase both the computing power and storage and the bandwidth of communication channels. Within the framework of this architecture, models were also proposed for the rational distribution of computing resources and memory, for example, in $[44,45]$.

However, for more rational use of AzScienceNet resources, taking into account the above, it is proposed to use the architecture of decentralized processing of scientific data in a network environment as in Fig. 4.

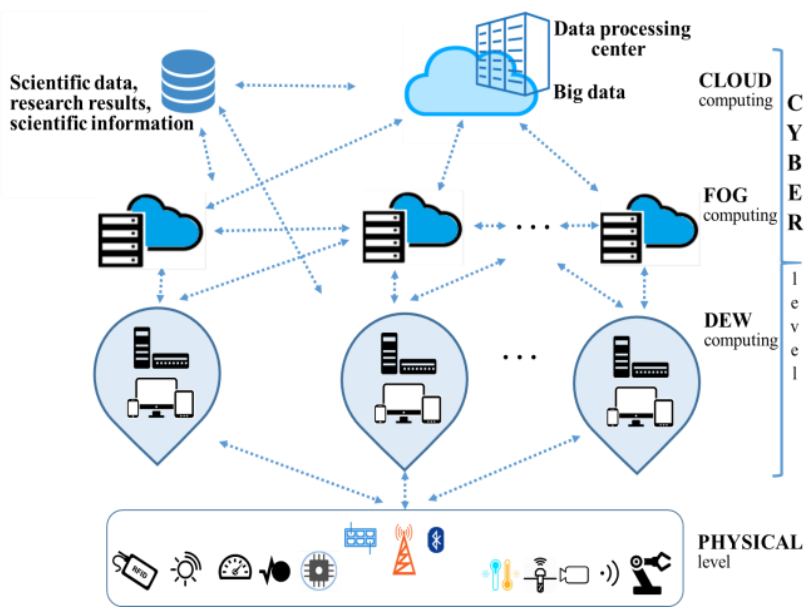

Fig.4. The generalized architecture of decentralized processing of scientific data.

As seen in Fig. 4, in integrating the CPS into the escience environment, an important factor is a physical level, at which scientific experiments are conducted and a large flow of scientific data is generated. Experiments are conducted on scientific profiles and spatially separated (Fig.1). Data is processed directly in dew computing clusters.

The proposed model of decentralized processing could be used, for example, in a system of round-the-clock monitoring of the stress-strain state of the earth's crust in seismogenic zones. At present, these studies have been carried out using a hand-held proton magnetometer at 70 rigidly fixed points [46]. A large stream of raw data should be collected at a central location for further processing. Thus, the main process is time-consuming and does not allow for optimizing the time frame for effective warning of natural disasters.

\section{CONCLUSION}

This article discusses the current trends in the integration of CPS into the e-science environment. This integration encompasses all phases from research data collection, storage, processing, and analysis, as well as 
science management problems. In addition, the e-science infrastructure information generated by CPS can be used for other purposes, such as uninterrupted power supply, materials, and equipment management, maintenance planning and optimized management to achieve higher overall performance and reliability of the e-science environment. considered in the context of a smart city. As an alternative to the centralized principle of organizing data processing, the prospects for decentralized data processing were presented and the possibilities of using the cloud, fog, dew and blockchain technologies for this purpose were considered. Decentralized computing covers a wide range of technical problems in the field of e-science, including equipment, operating systems, networks, databases, browsers, servers, etc.

In the future, practical works are planned on the integration of CPS in the environment of e-science in the framework of the solutions studied and presented in this article.

\section{REFERENCES}

[1] R. M. Alguliyev, R. G. Alakbarov, T. Kh. Fataliyev, "Electronic science: current status, problems and perspectives," Problems of information technology, 2015, No. 2, pp. 4-14. DOI: 10.25045/jpit.v06.i2.01.

[2] V. Gunes, et al., "A survey on concepts, applications, and challenges in cyber-physical systems," KSII Transactions on Internet and Information Systems, 2014, vol. 8, No. 12, pp. $4242-4268$.

[3] E. A. Lee, "Cyber-physical systems: Design challenges," 11th IEEE international symposium on object-oriented real-time distributed computing, 2008, pp. 363-369.

[4] E. A. Lee, and S. A. Seshia, Introduction to embedded systems: A cyber-physical systems approach. MIT Press, 2016.

[5] O. Cardin, "Classification of cyber-physical production systems applications: Proposition of an analysis framework," Computers in Industry, Elsevier, 2019, 104, pp.11 - 21. DOI: 10.1016/j.compind.2018.10.002.

[6] C. Greer, Cyber-Physical Systems and Internet of Things, NIST Special Publication 1900-202, 2019, p. 52.

[7] M. Chen, J. Wan, F. Li, "Machine-to-machine communications: Architectures, standards and applications," KSII transactions on internet and information systems, vol. 6, No. 2, 2012, pp. 480-497.

[8] T. Kh. Fataliyev, Sh. A. Mehdiyev. "Analysis and New Approaches to the Solution of Problems of Operation of Oil and Gas Complex as Cyber-Physical System," International Journal of Information Technology and Computer Science (IJITCS), 2018, vol.10, No.11, pp.6776, 2018. DOI: 10.5815/ijitcs.2018.11.07.

[9] A. Zanni. "Cyber-physical systems and smart cities," IBM Big data and analytics, 2015, vol. 20, pp. 1-8.

[10] R. Alguliyev, T. Fataliyev, and Sh. Mehdiyev, "Some issues of application of internet of things in the oil and gas complex," 6th International Conference on Control and Optimization with Industrial Applications, 2018, vol.1, pp. 46-48.

[11] S. K. Khaitan, and J. D. McCalley, "Design techniques and applications of cyber-physical systems: A survey," IEEE Systems Journal, vol. 9, no. 2, 2014, pp. 350-365.

[12] S. H. Ahmed, G. Kim, and D. Kim, "Cyber-Physical System: Architecture, Applications and Research
Challenges," 2013 IFIP Wireless Days (WD). IEEE, 2013.pp 1-5.

[13] N. Dey, et al., "Medical cyber-physical systems: A survey," Journal of medical systems, vol. 42, No. 4, 2018, p. 74.

[14] P. Hehenberger, et al., "Design, modeling, simulation and integration of cyber-physical systems: Methods and applications," Computers in Industry, vol 82, 2016, pp. 273-279. doi:10.1016/j.compind.2016.05.006.

[15] X. Liang, H. Chen, "The application of CPS in library management: a survey," Library Hi Tech, 2018, DOI: 10.1108/LHT-11-2017-0234.

[16] G. Werner-Allen, et al., "Fidelity and yield in a volcano monitoring sensor network," Proceedings of the 7th symposium on Operating systems design and implementation. USENIX Association, 2006, pp. 381-396.

[17] M. M. Rathore, et al., "Real-Time Big Data Analytical Architecture for Remote Sensing Application," IEEE Journal of Selected Topics in Applied Earth Observations and Remote Sensing, 2015, vol. 10, No. 8, pp. 4610-4621.

[18] G. Mois, S. Teodora, and C. F. Silviu, "A cyber-physical system for environmental monitoring," IEEE Transactions on Instrumentation and Measurement, 2016, vol. 65, No.6, pp. 1463-1471. DOI: 10.1109/TIM.2016.2526669

[19] V. A. Onufriev, A. S. Sulerova, and V. V. Potekhin, "Cyber-physical systems application for the radio telescope's adaptive surface control task," Symp. on Automated Systems and Technologies. Hannover: PZH Verlag. 2016, pp. 51-56.

[20] R. A. Langmann, "A CPS Integration Platform as a Framework for Generic Remote Labs in Automation Engineering," Cyber-Physical Laboratories in Engineering and Science Education, 2018, pp. 305-329.

[21] T. Kh. Fataliyev, Sh. A. Mehdiyev, "Problems of organization of e-maintenance in a network environment (in azerb.)," I republic scientific-practical conference, of actual problems of software engineering, Baku, 2017, pp. 291-293.

[22] H. S. Ning, H. Liu, "Cyber-physical-social-thinking space-based science and technology framework for the Internet of Things," Science China Information Sciences, 2015, vol. 58, No. 3, pp. 1-19.

[23] E. Hatziminaoglou, "Virtual observatory: science capabilities and scientific results," arXiv preprint arXiv: 0911.1878. 2009, Nov 10.

[24] P. Padher, V. M. Rohokale, "A Cyber-Physical System for Environmental Monitoring," Int J Sensor Networks and Data Communications, 2018, vol.7, issue 2, pp. 154-158. DOI: $10.4172 / 2090-4886.1000154$.

[25] L. Parolini, et al., "A cyber-physical systems approach to data center modeling and control for energy efficiency," Proceedings of the IEEE, vol. 100, no.1, 2011, pp. 254268.

[26] T. Nam, T. A. Pardo, "Conceptualizing smart city with dimensions of technology, people, and institutions," Proceedings of the 12th annual international digital government research conference: digital government innovation in challenging times, ACM, 2011, pp. 282-291.

[27] V. Albino, U. Berardi, and R. M. Dangelico, "Smart Cities: Definitions, Dimensions, Performance, and Initiatives," Journal of Urban Technology, 2015, Vol. 22, No. 1, 3-21.

[28] "Improving Performance with Integrated Smart Buildings," http://www.usa.siemens.com/intelligentinfrastructure/assets/pdf/smart-building-white-paper.pdf 
[29] R. Atat, et. al., "Big data meet cyber-physical systems: A panoramic survey," IEEE Access, 2018, vol. 6, pp. 7360373636. DOI: 10.1109/ACCESS.2018.2878681.

[30] Y. Liu, et al., "Review on cyber-physical systems," IEEE/CAA Journal of Automatica Sinica, vol. 4, No. 1, 2017, pp. 27-40.

[31] E. Althagafy, M .R. J. Qureshi, "Novel Cloud Architecture to Decrease Problems Related to Big Data," International Journal of Computer Network and Information Security (IJCNIS), Vol.9, No.2, pp.53-60, 2017. DOI: 10.5815/ijcnis.2017.02.07.

[32] R. G. Alakbarov, F. H. Pashaev, O. R. Alakbarov, "Forecasting Cloudlet Development on Mobile Computing Clouds," International Journal of Information Technology and Computer Science (IJITCS), 2017, vol. 9, No. 11, pp.23-34. DOI: 10.5815/ijitcs.2017.11.03.

[33] E. S. Jung, R. Kettimuthu, "Challenges and opportunities for data-intensive computing in the cloud," IEEE Computer, 2014, vol. 47, No 12, pp. 82-85.

[34] D. Linthicum. "Edge Computing vs. Fog Computing: Definitions and Enterprise Uses," 2018. Online: www.cisco.com/c/en/us/solutions/enterprisenetworks/edge -computing.html

[35] F. Bonomi, et al., "Fog computing and its role in the internet of things," Proceedings of the first edition of the MCC workshop on Mobile cloud computing, 2012, pp. 1316.

[36] Y. Wang. "Definition and Categorization of Dew Computing," Open Journal of Cloud Computing (OJCC), 2016, vol. 3, issue 1, pp. 1-7.

[37] Y. Wang, "Cloud-dew architecture," International Journal of Cloud Computing, 2015, vol. 4, No. 3, pp. 199210.

[38] Z. Zheng, et al., Blockchain challenges and opportunities: a survey, Work Pap. 2016. pp. 1-25

[39] R. S. Abdullah, M. A. Faizal, "BlockChain: Cryptographic Method in Fourth Industrial Revolution," International Journal of Computer Network and Information Security (IJCNIS), Vol.10, No.11, pp.9-17, 2018. DOI: $10.5815 /$ ijcnis.2018.11.02

[40] J. Lee, M. Azamfar, J. Singh, "A Blockchain-Enabled Cyber-Physical System Architecture for Industry 4.0 Manufacturing Systems," Manufacturing Letters, 2019. doi: https://doi.org/10.1016/j.mfglet.2019.05.003

[41] J. Van Rossum, Blockchain for research: Perspectives on a new paradigm for scholarly communication, Digital Science, November 2017.
[42] S. Bartling, "Blockchain for science and knowledge creation," Gesundheit digital, Springer, Berlin, Heidelberg, 2019, pp.159-180.

[43] https://azsciencenet.az/en/service/1

[44] R. Alakbarov, F. Pashayev, M. Hashimov, "A Model of Computational Resources Distribution among Data Center Users," IJACT, 2015, vol. 7, No. 2, pp. 01-06.

[45] R. G. Alakbarov, F. H. Pashaev, M. A. Hashimov, "Development of the Model of Dynamic Storage Distribution in Data Processing Centers," International Journal of Information Technology and Computer Science (IJITCS), 2015, vol.7, no.5, pp.18-24. DOI: 10.5815/ijitcs.2015.05.03.

[46] A. G. Rzayev, et al "Reflection of the geodynamic regime of the Shamakhi-Ismayilli seismogenic zone in local anomalies of the geomagnetic field," Seismoprognosis observations in the territory of Azerbaijan, 2019, vol. 16, No. 1, pp.7-16.

\section{Authors' Profiles}

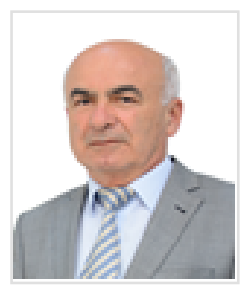

Tahmasib Khanahmad Fataliyev graduated from Automation and Computer Engineering faculty of Azerbaijan Polytechnic University. His primary research interests include various areas in e-science, data processing and computer networks.

$\mathrm{He}$ is head of the department at the Institute of Information Technology of ANAS, Azerbaijan. He is the author of about 120 scientific papers.

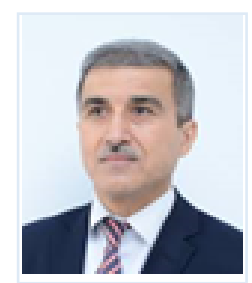

Shakir Agajan Mehdiyev graduated from Automation and Computer Engineering faculty of Azerbaijan Polytechnic University. His primary research interests include various areas in e-science, computer networks, and maintenance.

$\mathrm{He}$ is head of the department at the Institute of Information Technology of ANAS, Azerbaijan. He is the author of about 25 scientific papers.

How to cite this paper: Tahmasib Kh. Fataliyev, Shakir A. Mehdiyev, "Integration of Cyber-Physical Systems in EScience Environment: State-of-the-Art, Problems and Effective Solutions", International Journal of Modern Education and Computer Science(IJMECS), Vol.11, No.9, pp. 35-43, 2019.DOI: 10.5815/ijmecs.2019.09.04 